\title{
Trend Topics and Changing Concepts of Computational Design in the Last 16 Years
}

\section{A content analysis}

\author{
Orkan Zeynel Güzelci ${ }^{1}$, Sema Alaçam ${ }^{2}$, Handan Güzelci ${ }^{3}$ \\ ${ }^{1,3}{ }_{\text {Istanbul Kultur University }}{ }^{2} \dot{I}_{\text {stanbul Technical University }}$ \\ 1,2\{orkanguzelci|semosphere\}@gmail.com³h.duyar@iku.edu.tr
}

This study argues that analysis of written content might be helpful to provide clues at a certain extent on the future directions of current research areas and the emergence of new study areas. In the scope of the study, the International Journal of Architectural Computing (IJAC) which has been a scientific platform covering many pioneer publications on education research in computer-aided architectural design (CAAD) field was selected as source content. Although the size of the source domain is limited, the analysis of abstracts and titles of 439 articles published in IJAC between 2003 and 2018 revealed promising results which can be examined under four characteristics: "constant", "emerging", "fading" and "solidifying" concepts. The tokens in the analysis process are words, phrases, topic nodes and links between topic nodes. The outcomes of this study might contribute to tracking the evolution of concepts their emergence or disusage in different time and contexts, and interrelations between different concepts.

Keywords: content analysis, computational design concepts, IJAC

\section{INTRODUCTION}

The reflection of digital technologies on theory and practice of architectural design has been discussed over the last two decades. Widespread usage of personal computers, changes in the way the architectural knowledge is represented, decoded, reproduced and shared through digital media have been on the way architects think and design. Not only the hierarchical organization of the architectural offices but also the terminology, concepts, and description of methodologies have been evolving relatedly. With the impact of technological changes, the transformation in architectural design practice has led to the emergence of new concepts, and some of the existing concepts have started to convey new meanings. In that sense, spoken or written language has the potential to provide indicators about the direction of changes and transformations. The indicators which can be captured from discourse should be considered as an anchor or focal points of transformation, which do not cover all aspects and dimensions of the technological transformation in architectural design practice.

This study aims to provide an interrelation map on the computation concepts reflected into the architectural design field, through analysing keywords, 
concepts, and phrases in the published media. As de Sola Pool (1959) underlines the limitations of wordbased content analysis in the extraction of meanings of any context, it might still have the potential to provide insights on cause-effect relationships. Taking the difficulties of content analysis method in mind, the motivation of the study is derived from gaining more insights into the potential patterns embedded in the changing concepts of computational design domain. The main elements of the pattern searching process are considered as words, phrases, topic nodes, and links among topic nodes. Based on these assumptions, this study aims to investigate whether it is possible:

- to extract trend topics based on the frequency of the words and phrases;

- to find distinctive characteristics of topic nodes;

- to make predictions on the direction of change and characteristics of topic nodes;

- to make inference about the emergence of new methods, tools, approaches and their reflection onto scientific studies based on the content analysis of a given text.

In the scope of this study, the abstracts and the titles of the peer-review articles in the International Journal of Architectural Computing (IJAC) between 2003 and 2018 were used as a source of data. The abstract of 439 articles in 63 issues which were published in a 16 years period was analysed. Although there have been various conferences on CAAD which made it possible for the people from universities, practice and industry to encounter and gather since 1960s (Celani and Veloso, 2015), accumulating the experience of sister conferences such as eCAADe, ACADIA, SIGraDi, ASCAAD, CAADRIA, and CAAD became a distinctive advantage for IJAC to provide a common ground for opening new debates in theory and practice. This study introduces a content analysis method to be used in the analysis of concepts and trend topics of CAAD. Four main characteristics are explored in the initial analysis process: constant, emerging, fad- ing and solidifying concepts. Further to the association of the explored characteristics with time and frequency values, it is observed that the proposed method has potential to become an analysis tool providing a basis for different readings of the given text and contexts.

\section{RELATED STUDIES}

The term discourse is defined as "a particular way of talking about and understanding the world or an aspect of the world" by Philips and Jørgensen (2002). As an umbrella term, the discourse has been approached by people from various fields such as philosophy, psychology, sociology, linguistic or political science in different axes and extends. As a common denominator, discourse analysis refers to the analysis of language or language-like structures to extract new relations, patterns or frames (Philips and Jørgensen, 2002). Relational topic models are wellestablished methods (Chang and Blei, 2009; Marcin and Shiu, 2012) both analyzing a larger amount of data and extracting relations from the source content. Another approach, the critical analysis concerns the demystification of social context through using written or spoken language. Regarding the reflection of technological advances into architectural practice and discourse, what one calls novel easily becomes common and acquaintance in a short period of time. On one hand, new concepts have been emerging, on the other hand as a living organism the meaning and impact area of an existing concept is being transformed continuously.

In relation to the concepts, topics, and approaches of computer-aided architectural design (CAAD), remarkable literature has started to be accumulated with an increasing acceleration since the early 1990s. Apart from the widespread use of digital technologies in architectural design, the written culture involving books, conference papers, and articles have also contributed to expanding the knowledge in the digital design field. As an instance, the web-based database, Cumulative Index of CAAD (CUMINCAD.SciX.net) has provided a common plat- 
Figure 1

Flowchart of the proposed methodology form for academicians, practitioners, and students since 1998. When it came to 2003, in the second issue of IJAC, Martens and Turk mentioned the necessity of a systematic approach to categorize, store and recall the information in the growing body of CUMINCAD database. Martens and Turk (2003) provided a provisional list of 30 keywords to be used in indexing the CAAD related concepts. Celani and Veloso (2015) presented a comprehensive overview of the history of CAAD, tracing back to the theoretical studies in the 1960s and focusing on the conferences between 1980s and 2015. Investigating the trends, topics, concepts of conferences related to CAAD, Celani and Veloso (2015) introduced the most frequent keyword index used in CUMINCAD database. Liu et al. (2017) presented a domain-specific analysis of BIM concepts in bibliographic records and their evolution, examining 1874 BIM-related articles. Liu et al. (2017) unfolded thresholds and periods and their relationship with BIM-related concepts. Turk and Cerovšek (2003) underlined the similarities between computer-based understanding of two-words phrase selection and human intervention-based selection approach and suggested machine learning techniques as a leading future direction. At some extent, this study can be considered as an attempt to revisit the topic map of Turk and Cerovšek (2003), based on an extended and updated source of data. In addition, it will provide a comparison opportunity between Martens and Turk (2003)'s provisional list published in the second issue of IJAC and the new list extracted from IJAC database.

\section{METHODOLOGY}

The proposed content analysis method involves four steps (Figure 1): (i) quantitative analysis (frequency of word and phrase), (ii) frequency mapping (yearphrase correlation), (iii) qualitative analysis (characteristics of concepts), (iv) relational mapping (topic nodes and links).

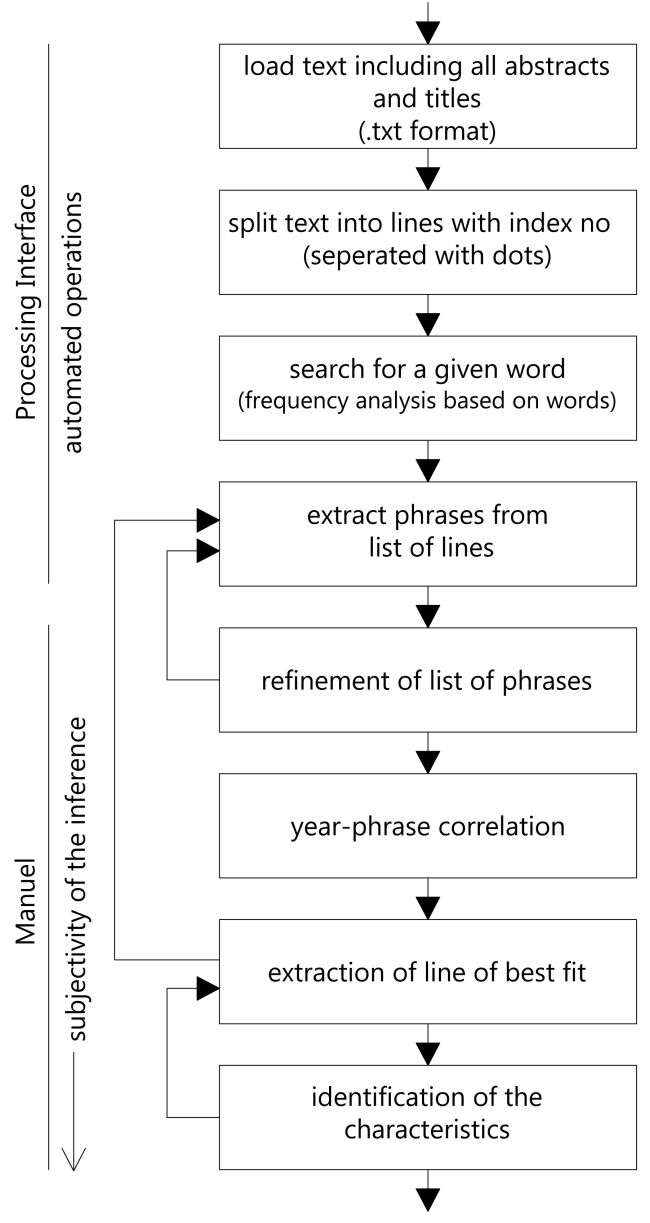

Apart from the list of 30 keywords derived from CUMINCAD database presented by Martens and Turk (2003), 27 keywords were given by Celani and Veloso (2015) as the most frequent keywords found in CUMINCAD's keyword index. In the qualitative analysis process, not only Martens and Turk (2003)'s and Celani and Veloso (2015)'s keywords were used but also new words are added by the authors. As a result of human intervention based and computer-based word extraction processes (Figure 2; Figure 3), 60 key- 
words are chosen to be investigated in further steps.

\begin{tabular}{|c|c|c|}
\hline \multicolumn{2}{|c|}{ list of keywords taken from the literature } & \multirow{2}{*}{$\begin{array}{c}\text { frequency based selection } \\
\text { IAC } \\
2003-2018\end{array}$} \\
\hline $\begin{array}{c}\text { Martens \& Turk } \\
2003\end{array}$ & $\begin{array}{c}\text { Celani \& Veloso } \\
2015\end{array}$ & \\
\hline$n_{1}=30$ & $\mathrm{n}_{2}=27$ & $\mathrm{n}_{3}=60$ \\
\hline \multicolumn{3}{|c|}{ O k: number of overlapping keywords } \\
\hline
\end{tabular}

The quantitative analysis process is simply based on word and phrase extraction from the source content depending on their frequency. Further to machineassisted word extraction (Url-1), Processing interface was used for more precise calculations. The source content is titles and abstracts of the selected 439 articles which were published in IJAC between 2003 and 2018. Frequency mapping is the scatter plot representation of phrases in year ( $x$ ) and frequency $(y)$ axis. The third step use frequency mapping as a layout to determine trend lines (line of best fit) (Figure 4).

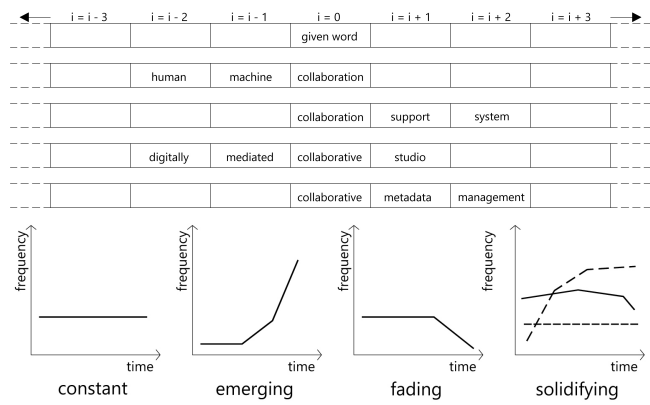

However, involving human intervention, the qualitative analysis can be considered more subjective or intuitive in comparison to the other steps. Four trend lines (constant, emerging, fading, solidifying) are selected as the main characteristics of concepts. Further to the detection of content-dependent trend lines, characteristics of each phrase were examined and listed. In the fourth step, Chang and Blei (2009)'s relational topic models were utilized, however differ- ent than their model, the mapping process was made manually for the selected phrases (Figure 5).

The initial results show that there might be an accumulation in the usage of specific keywords. This information is not enough to explain certain tendencies and popularization of the concepts; however, frequency mapping still provides insights into the emergence and evolution of the concepts. The limited analysis of the selected keywords provided insights on the existence, usage, and transformation of the concepts in different domains. In this process, Turk and Cerovšek (2003)'s topic mapping technique which was used for extracting 2-word phrases were used and further it was adapted via a subjective selection of the authors. Different than the topic mapping technique, apart from the most frequent terms also some terms which are less frequent but might be influential in long terms were kept.

\section{FINDINGS AND OUTCOMES}

While the first column of Figure 6 includes the list of keywords refined by Martens and Turk (2003), the second column involves the keywords suggested by Celani and Veloso (2015). It is observed that the frequency of the three-fold specified phrases introduced by Martens and Turk (2003) such as "case based reasoning", "computer integrated construction", "Object Oriented Modeling" in IJAC database is quite low. On the other hand, more generic keywords suggested by Celani and Veloso (2015) took higher frequency scores. Further to machine-assisted word extraction of IJAC database, 60 keywords are selected and listed in the third column of Figure 6 . In this step, as it is shown in Figure 2, the overlapping keywords among the first three columns of Figure 6 are merged. As a result of analyses based on 439 articles abstracts published in a 16 years period. During the initial data analysis, four prominent characteristics were observed:

- Constant concepts: Conveying the same meaning with minimum contextual change or without any change.

- Emerging concepts: It is related to how a
Figure 2

Calculating the overall number of keywords

Figure 3

Adjacency based phrase extraction from the string lines

Figure 4

The main characteristic trends appeared in the year-based phrase distribution graph 
Figure 5

Relational topic model based on the selected keywords and phrases concept has begun to become a field-specific term by time with an increasing acceleration in trend curve and with an increasing ability to derive new phrases. The parameters for this item are the year-based frequency, the frequency-based trend curves and the ability to derive new phases. The ability to derive new phrases is represented with the radius of the circles in Figure 7.

- Fading concepts: According to year-based frequency, the concepts which become less popular by time. The concepts which have a decreasing frequency-based trend curve.

- Solidifying concepts: It refers to the group of words which are transformed into fieldspecific phrases by time. However, different than "emerging concepts", the keywords stay the same without becoming a part of new phrases. In other words, solidifying concepts do not manifest the ability to derive new phrases.

Figure 7 contains the information of selected keywords, count of repetitions, derivation of phrases, seen years, and characteristics of concepts. In Figure 7a, if we take 2003-2018 scale into consideration, the line of the best fit of the keyword "collaborative/collaboration" indicates a "fading" characteristics. However, the keyword "collaborative/collaboration" (C) and "collaborative design" (CD) have been a part of various phrases such as $C D$ experience, $C D$ simulation game, $C$ Project, Distributed $C$ Architectural Virtual Environments, interactive $C D$ pedagogies, $C A R$, Distributed $C D$, voxel-based $C$ modelling, $C D$ research, $C$ metadata management, C networks, C Semantic Management, 3D C environment, $C D$ management, $C D$ processing, $C$ environment, Cyber-Enabled CD Studio, digitally mediated $C$ studio, Network-centric CD platform, $C$ architectural design, $C D$ environment, $C D$ practice, $C$ development, $\mathrm{C}$ robotics, $\mathrm{C}$ digital design experience. In that sense, "collaborative/collaboration" keyword performs a "derivative" property and the ability to derive new phrase has a stabilization at an extent. Therefore, the derivative property of "collaborative/collaboration" keyword is considered as "constant".

In Figure 7b, the year-based distribution graph of the keyword "learning" is shown. For the key-

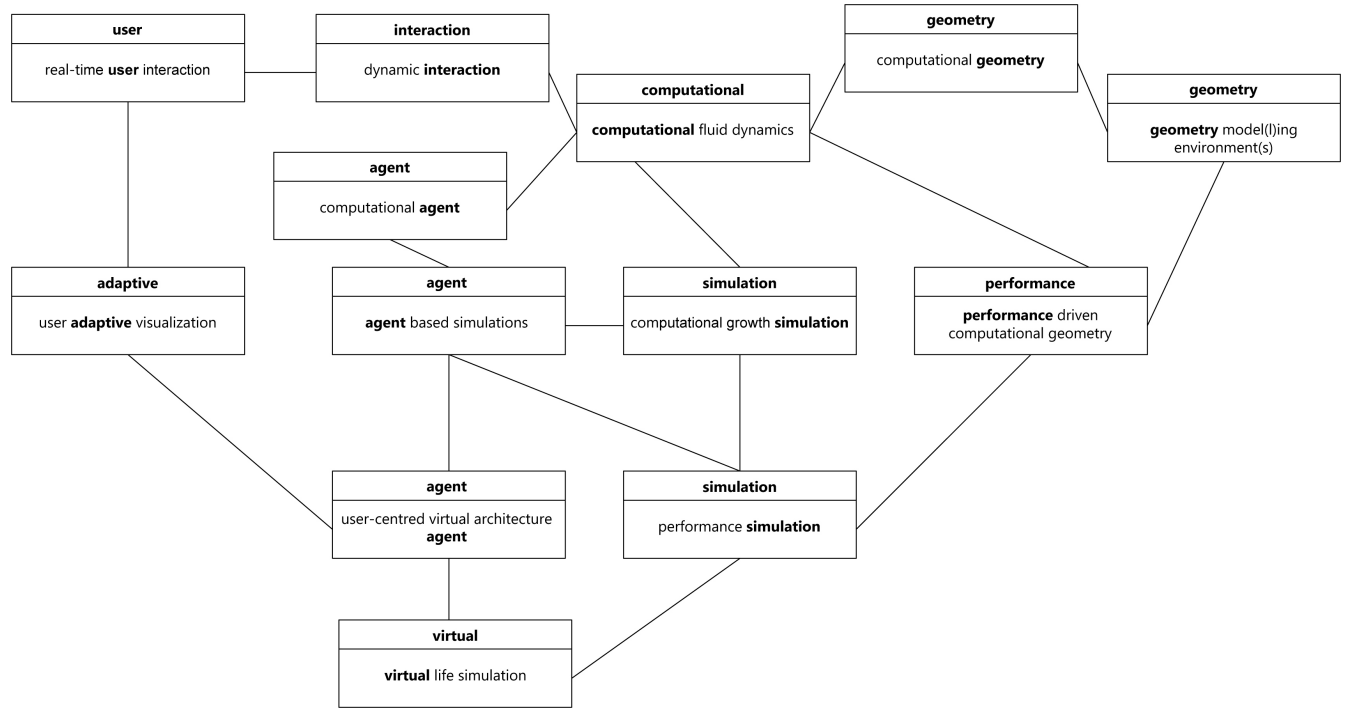




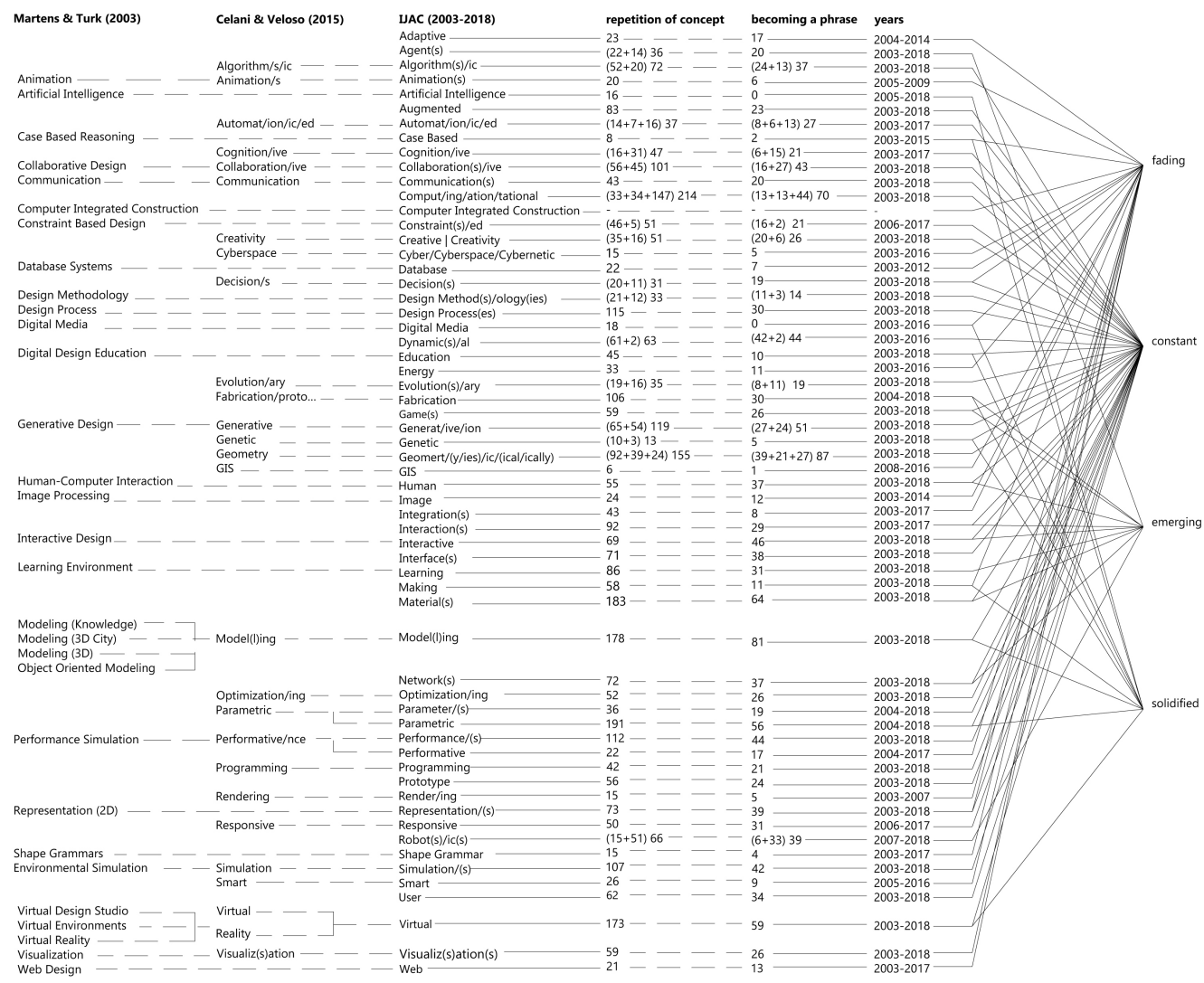

Figure 6

Comparative list of keywords and their features 
Figure 7

Samples of four main

characteristics.

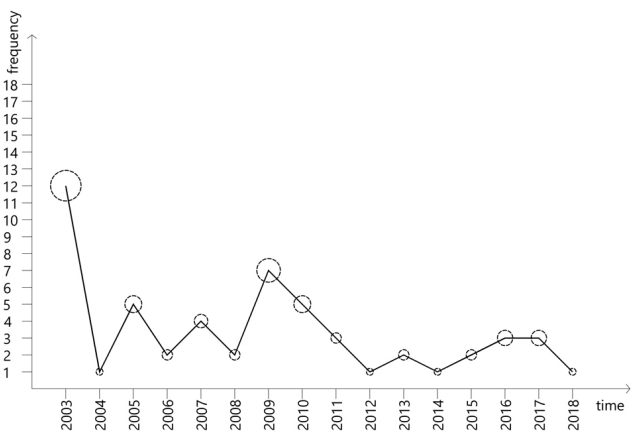

(a) word: collaborative/collaboration / characteristic of phrases: constant

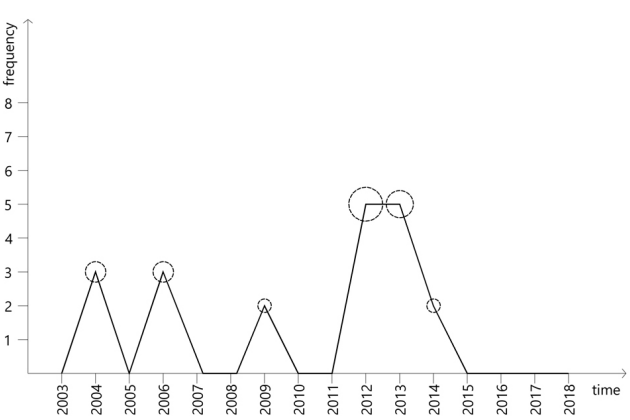

(c) word: adaptive / characteristic of phrases: fading

word "learning", the ability to derive new phrases has been distinctively higher in the years 2009, 2014 and 2018. The context, meaning and related concepts of "learning" differentiate dramatically such as "hands-on learning" (2003), "learning algorithms" (2004), "design learning" (2004) or Just-in-Place (JIP) learning (2014). On the other hand, "deep-learning" (2018), "deep learning algorithms" (2018), "functiondriven deep learning" (2018) constitute a "new" topic node which displays an "emerging" characteristics.

The keyword "adaptive" is shown in Figure 7c which reveals the popularity of the keyword continues until 2014 discreetly. There have been pick years as listed: Adaptive information, Adaptive vision, User adaptive visualization (2004); Adaptive architecture/s, Adaptive assemblies, Complex-adaptive

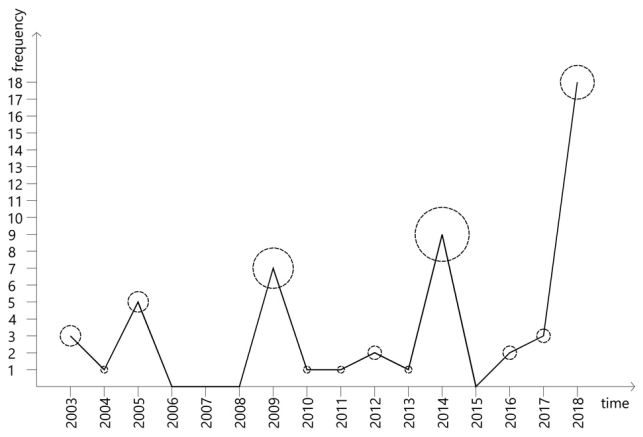

(b) word: learning / characteristic of phrases: emerging

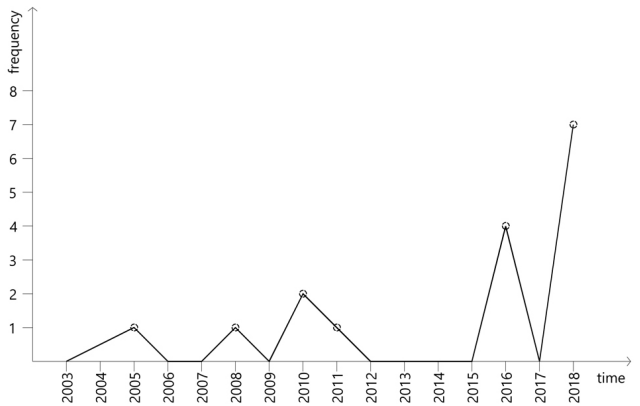

(d) word: artificial intelligence / characteristic of phrases: solidifying

framework (2006); Real-time adaptive building skin systems, Real-time adaptive intelligent building skin systems (2009); Adaptive artificial lighting element, Adaptive design, Adaptive distributed robotics, Adaptive solar envelope (ASE) (2012); Adaptive hybrid structure, Adaptive mechanisms, Adaptive structure (2013); Adaptive liquid lens, Adaptive natural light spotlights (2014). The adaptive keyword is considered as a "fading" phrase, which at the same time it has been consisting of more complex phrases (engaging with more specific context) between 20122014.

Another example, the frequency-year graph of "artificial intelligence" is shown in Figure 7d. Having a solidifying characteristic, the term has been used as it is between 2003 and 2018. In other words, the num- 
ber of the keyword seen increased however it does not carry a derivative characteristic.

\section{DISCUSSION}

As words and phrases with solidifying characteristics have become part of the design discipline, they cannot be ignored in digital design education. According to the findings of the study, it is observed that the words and phrases which have solidifying characteristics are used as tools or methods in architectural practice and education. The teaching and use of these tools and methods might be suitable for use in undergraduate education as they become understandable with the help of studies at a certain level.

The outcomes of this study might contribute to tracking the evolution of concepts their emergence or disuse in different time and contexts, and interrelations between different concepts. It is planned to extend the data source analysed in future studies in order to gain more consistent results. Despite the limitations of the outcomes of the study, it is still possible to speculate on the following issues. However, it should be kept in mind that an investigation of a larger amount of data might affect the results.

The trend curve of the "emerging" keywords displays an exponential growth and emerging keywords do not have a direct connection with the "tools". Being closer to the methods and approaches, the "emerging" keywords have the potential to reflect onto and transform the architectural curricula in the longer term. Due to its floating ground of meaning, it is thought that emerging keywords might be more influential in the graduate levels in the short term.

Another interesting finding is that the same keywords might convey more than one characteristic when we consider different timespans. Therefore, the scope, timespan, amount of source content are crucial indicators of the analysis process. This study can be considered relatively closer to the symbolic methods of language theories; however, in future studies, it is planned to elaborate the study through using stochastic Bayesian analysis methods. The selected 60 keywords will be used for further analysis based on four assumed elaborate the discussions on the reflection of tools, medium, techniques into the architectural design domain, and the existing concepts which gain new meanings.

\section{REFERENCES}

Celani, G and Veloso, P 2015 'CAAD conferences: A brief history. In The next city-New technologies and the future of the built environment', Proceeding of CAAD Futures 2015, São Paulo, pp. 47-58

Chang, J and Blei, D 2009 'Relational topic models for document networks', Proceedings of International Conference on Artificial Intelligence and Statistics (AISTATS) 2009, Florida, USA, pp. 81-88

$\mathrm{Li}, \mathrm{X}$, Wu, P, Shen, GQ, Wang, $\mathrm{X}$ and Teng, Y 2017, 'Mapping the knowledge domains of Building Information Modeling (BIM): A bibliometric approach', Automation in Construction, 84, pp. 195-206

Marcin, I and Shiu, S 2012, Extracting Topic Trends and Connections: Semantic Analysis and Topic Linking in Twitter and Wikipedia Datasets, Final Project, Stanford University

Martens, B and Turk, Z 2003, 'Cumulative index of CAAD: current status and future directions', International Journal of Architectural Computing, 1(2), pp. 219-231

Phillips, L and Jørgensen, MW 2002, Discourse analysis as theory and method, Sage Publications

Pool, IDS 1959, Trends in content analysis, University of Illinois Press.

Turk, Ž and Cerovšek, T 2003 'Mapping the W78 Papers onto the Construction Informatics Topic Map', Proceedings of the 20th CIB W78 Conference on Information Technology in Construction, pp. 423-431

[1] https://www.databasic.io/en/wordcounter/

[2] http://journals.sagepub.com/home/jaca 